\title{
Neutron skins from Coherent Pion Photoproduction
}

\author{
Lorenzo Zana for the A2 collaboration at MAMI ${ }^{1}$, a \\ ${ }^{1}$ University of Edinburgh, James Clerk Maxwell Building, Peter Guthrie Tait Road, Edinburgh, EH9 3FD, UK.
}

\begin{abstract}
These proceedings outline the new program of neutron skin measurements utilising coherent pion photoproduction from nuclei. The experimental program utilizes the Crystal Ball and TAPS detector systems at the tagged photon beam facility at MAMI. Recent results from the program and future plans are presented.
\end{abstract}

\section{Introduction}

The coherent production of $\pi^{0}$ mesons from nuclei is a promising method to extract information on the size and nature of neutron skins in nuclei. The neutron skin thickness $\left(\Delta r_{n p}\right)$, defined as the difference between the root mean square (rms) radii for the neutron and proton distributions, are predicted to form on heavy nuclei having a neutron excess [2-4]. A detailed and accurate understanding of the properties of neutron skins would provide valuable constraint on nuclear theories [6-13, 15-19, 21, 22] which predict a wide range of neutron skin thicknesses, despite being constrained by the wealth of data on other nuclear properties. Additionally, information on the equation of state (EOS) for neutron rich matter can be extracted from neutron skin measurements, due to the tight correlation between the size of the neutron skin and the magnitude of poorly established parameters of the EOS. Most importantly the neutron skin measurements offer the opportunity to better constrain the density dependence of the symmetry energy for the EOS for neutron rich matter. The poor knowledge of this quantity has consequences for our understanding of basic properties of neutron stars, including mass-radii relationships and possible cooling mechanisms.

For incident photon energies where the excitation of the $\Delta(1232)$ resonance dominates the coherent $\pi^{\circ}$ reaction process proceeds with a close to equal probability on protons and neutrons. For these regions access to information on the matter form factor with an electromagnetic probe can be obtained. The photon is a close to ideal probe for nuclear structure studies. It does not undergo strong interactions in the entrance channel, which have complicated the interpretation of data using strongly interacting probes e.g. proton scattering. The contribution of Coulomb interactions in the entrance and exit channels in coherent $\pi^{0}$ photoproduction is also negligible. These are both important advantages when accurate nuclear structure studies of large, highly charged atomic nuclei are required. However, the effect of the final state $\pi^{0}-A$ interaction must

\footnotetext{
ae-mail: lzana@ph.ed.ac.uk
}

be accounted for in the theoretical model. The effect of $\pi^{0}-A$ interactions on the measured data is minimized by choosing low pion momenta where this interaction is relatively weak. Measurement over a wide range of momentum transfer and pion momenta allow its treatment in the theoretical model to be assessed.

In these proceedings are presented recent measurements and future plans for coherent pion photoproduction from nuclei using the Crystal Ball and TAPS detectors at the MAMI beam facility in Mainz, Germany. These experiments achieve the statistical and systematic precision necessary to infer for the first time an accurate information on the neutron skin of nuclei from this reaction. Section 2 outlines the experimental facilities used in the program. Section 3 shows the recent results on the skin for ${ }^{208} \mathrm{~Pb}$ and outlines recent further measurements and the plans for future.

\section{Experimental apparatus}

The experimental apparati used in the measurement are described in the following.

\subsection{The MAMI-C accelerator facility}

The MAMI-C[25] electron beam facility produces a 1.65 $\mathrm{GeV}$ high quality $100 \%$ duty factor electron beam from a series of 4 cascaded racetrack microtrons. The most recent of these, a harmonic double sided microtron was installed in 2005 .

The MAMI-C electron beam can be passed to 4 experimental halls. The Crystal Ball[23] and TAPS[24] calorimeters are used in the real photon hall (A2 on Figure 1). In this hall the electron beam is converted to an intense $\left(10^{8} \gamma \sec ^{-1}\right)$ beam of real photons through bremsstrahlung on a thin metal foil radiator. The scattered electrons in this process are momentum analyzed in the Glasgow/Mainz Tagger, a magnetic spectrometer[27] which provides a determination of the energy of the associated bremsstrahlung photon with a resolution of 2-3 MeV. Improved resolution 


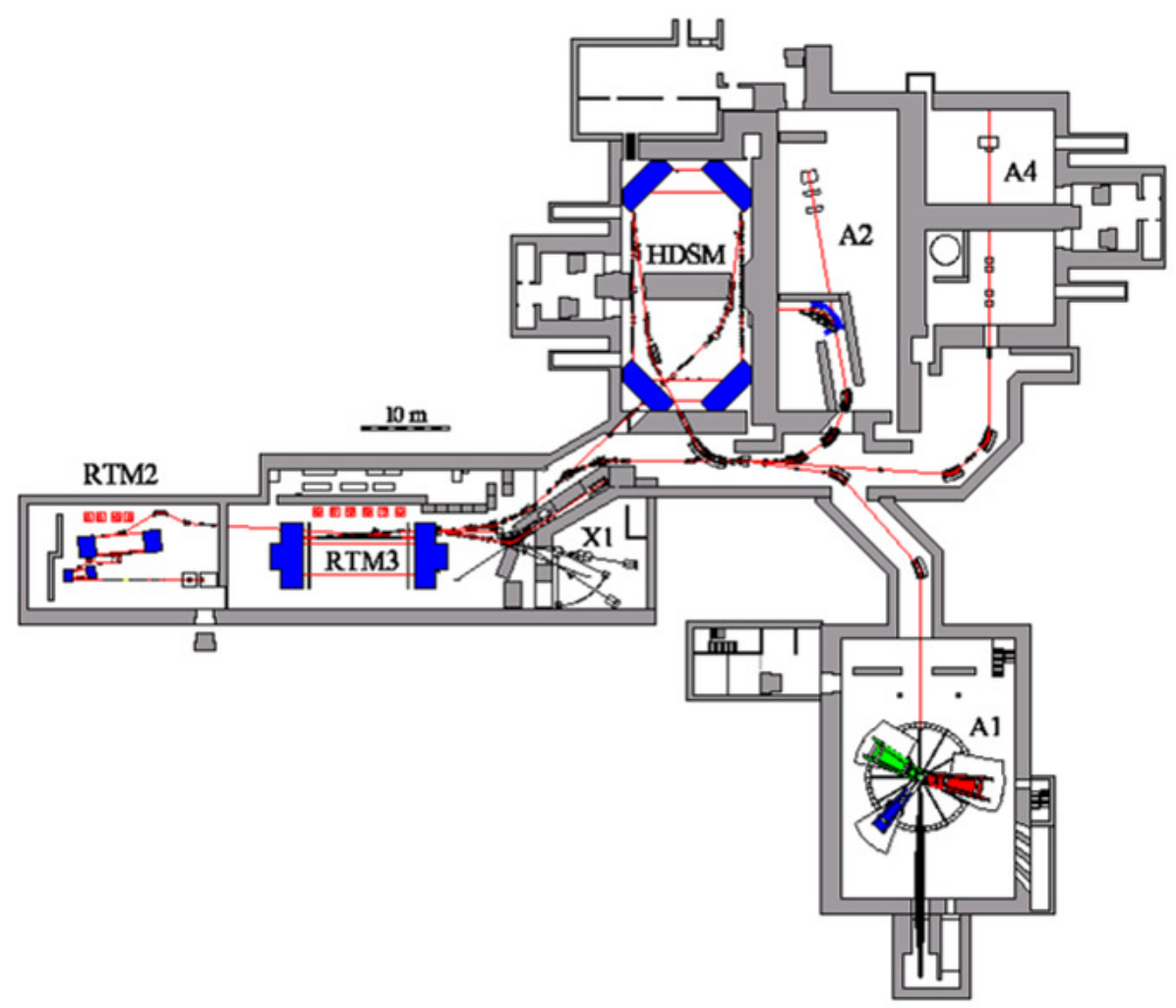

Figure 1. Layout of the experimental halls at the MAMI accelerator facility. The real photon beam experiments are carried out in the A2 hall

and rate can be achieved for smaller sub ranges of photon energy by using a more highly segmented focal plane microscope sub-detector system [28]. To extend the upper energy range of the tagged photons a new endpoint tagger has just been successfully commissioned at the facility which tags photons up to $\sim 93 \%$ of the incident electron beam energy. The photon beam can be produced with a high degree of linear $(75 \%)$ or circular $(85 \%)$ photon polarization.

\subsection{Crystal Ball}

The Crystal Ball was conceived in the mid 1970s at SLAC and has been involved in experiments at SPEAR, DORIS and Brookhaven National Laboratory before arriving at MAMI. The Crystal Ball is a 672 element NaI detector covering $94 \%$ of $4 \pi$. Each element is shaped like a a truncated pyramid $41 \mathrm{~cm}$ long (Figure 2). Photons incident on the ball produce an electromagnetic shower which generally deposits energy in a number of crystals (98\% of the deposited energy of each photon is contained in a cluster of 13 crystals). From analyzing the centre of gravity of the shower angular resolutions for the photon of $2-3^{\circ}$ in theta and $2^{\circ} / \sin (\theta)$ in phi are achieved. The high light output of $\mathrm{NaI}$ also permits a good determination of the photon energy $(\sigma / \mathrm{E} \quad 1.7 \% / \mathrm{E}(\mathrm{GeV}) 0.4)$.

The scintillation light deposited in each crystal is read by its own 2 inch Photomultiplier tube (PMT). The output signal from each PMT is fed to a split-delay module.
One output branch gives analogue sums of 4 or 16 crystals which can be used in trigger decisions. The second output branch of the split feeds individual multi hit TDCs and scalers via a PM98 dual threshold discriminator. The third output branch is fed to a sampling ADC (i-SADC108032) which samples the pulse shape at $40 \mathrm{MHz}$. The ADC modules have an $80 \mathrm{MHz}$ sampling capability.

Although the full sample of the pulse shape can be digitized and read out into the data stream, currently only the integral of the sampled signal is recorded. This integrated signal is output for three time regions before, during and after the scintillation pulse. This enables correction for remnant light present in the crystals before the event of interest to be corrected for, which improves the energy resolution.

The controller boards for the ADC, TDC and scaler use the GeSiCA and CATCH electronics developed for the Compass experiment at CERN.

The photon calorimetry capabilities of the Crystal Ball have been well established. The detector has had a more limited history in use for hadron calorimetry, but the low energy final state particles produced in many reactions at the Mainz beam energies made the development of this capability extremely desirable. The length of the counters corresponds to the stopping range of $233 \mathrm{MeV}$ for charged pions, $341 \mathrm{MeV}$ for charged Kaons and $425 \mathrm{MeV}$ for protons.

For experiments at Mainz two sub-detector systems are placed inside the ball (Figure 2). The two multi wire pro- 


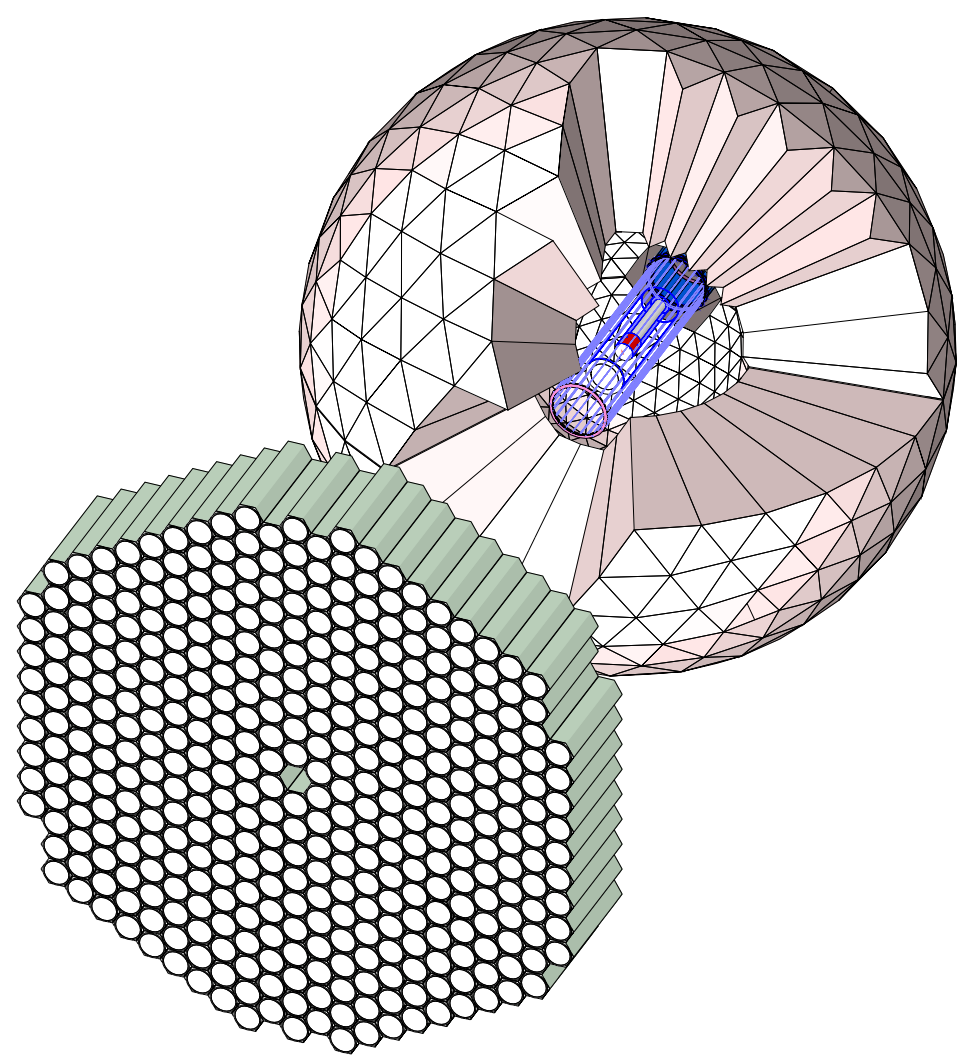

Figure 2. The CrystalBall, TAPS, MWPC, PID detectors

portional chambers (MWPCs) comprise 528 anode wires in the beam direction with 332 helically wound cathode strips. The detector was previously used with the Daphne detector[41]. The device gives accurate track information for charged particles with a resolution of $\sim 1^{\circ}$ in theta and $\sim 2^{\circ}$ in phi.

A 4mm thick segmented plastic scintillator barrel [37] forms the new particle identification detector (PID). The correlation of the energy deposit in the PID and the CB/TAPS systems allows the various charged particle types to be distinguished. Each scintillator element is $500 \mathrm{~mm}$ long with one side angled at 15 degrees so that the 24 scintillators can be formed into a barrel with minimal gaps between the scintillator edges. The scintillators are read out at one end by $9 \mathrm{~mm}$ diameter Hamamatsu Photonics R1635 photomultiplier tubes.

\subsection{TAPS BAF2 calorimeter}

The TAPS detector system covers the forward angle region for $\pm 20^{\circ}$ which is not covered by the Crystal Ball. This is an important part of the phase space for fixed target experiments with high beam momenta. TAPS consists of 384 hexagonally shaped $\mathrm{BaF} 2$ detectors each $25 \mathrm{~cm}$ long corresponding to 12 radiation lengths. TAPS is a versatile detector system and has been employed at MAMI, GSI,
Ganil, CERN and most recently with the Crystal Barrel at Bonn. $\mathrm{BaF}_{2}$ scintillator has two scintillation components, a fast component with a decay time of $0.6 \mathrm{~ns}$ and a slow component with a decay time of $620 \mathrm{~ns}$. The energy resolution for the detector is of similar order to that obtained in the Crystal Ball.

New VME based readout electronics for the TAPS detectors have been constructed[38] for it's use with the Crystal Ball. The signal feeds two leading edge discriminators (LED1,2), the outputs of which are used for flexible event and trigger selection. The constant fraction discriminator (CFD) gives an accurate timing for the pulse with reduced amplitude dependent jitter or walk. This accurate timing signal is necessary to gate the charge to analogue converters for the fast component of the scintillator response, which is only integrated for 20ns. The signal from the PMT is fed to 4 separate integration channels in which the fast and slow components of the scintillator response are measured with both low and high gain. This gives an accurate determination of the pulse shape over a wide dynamic range and conserves the excellent pulse shape discrimination capabilities of $\mathrm{BaF}_{2}$ scintillator. 


\section{Results and plans}

The Crystal Ball and TAPS detector offer a highly efficient and largely uniform detection efficiency for $\pi^{0}$ mesons. Previous $\left(\gamma, \pi^{0}\right)$ measurements for ${ }^{208} \mathrm{~Pb}[39,40]$ either did not use isotopically pure targets or did not achieve the precision needed to study the neutron skin, mainly because they used $\pi^{0}$ detection systems with limited angular coverage resulting in a small detection efficiency with too large a dependence on pion energy and angle to give definitive results.

\subsection{Recent results for ${ }^{208} \mathrm{~Pb}$}

Figure 3 shows the published ${ }^{208} \mathrm{~Pb}\left(\gamma, \pi^{0}\right)$ cross section data [30], presented as a function of momentum transfer, $q$. In order to extract information about the nucleon distribution in ${ }^{208} \mathrm{~Pb}$, the measured $\left(\gamma, \pi^{0}\right)$ cross sections are compared with predictions from the model of Drechsel $e t$. al. [42], which represents $\pi^{0}$ photoproduction using a unitary isobar model and includes a self-energy term for $\Delta$ propagation effects in the nucleus. The pion-nucleus interaction is treated using a complex optical potential [1], whose parameters are fixed by fits to pion-nucleus scattering data. In the $\left(\gamma, \pi^{0}\right)$ model the nucleon density distribution, $\rho(\mathrm{r})$, is parametrised as a single symmetrised two parameter Fermi distribution $(2 \mathrm{pF})$ [1] with half-height radius $c$ and diffuseness $a$. For the present analysis different proton and neutron distributions, each separately parametrised by a $2 \mathrm{pF}$ distribution are used to describe the nuclear shape $\rho(r)=(\mathrm{Z} / \mathrm{A}) \rho_{p}(r)+(\mathrm{N} / \mathrm{A}) \rho_{n}(r)$. Then in order to put $\rho(r)$ into the $\left(\gamma, \pi^{0}\right)$ code it is fitted by a single $2 \mathrm{pF}$ distribution [43]. The parameters for $\rho_{p}(r)$ are taken from electron scattering data [44], viz. $a_{p}=0.447 \mathrm{fm}$ and $c_{p}=6.680 \mathrm{fm}$, corrected for the finite size of the proton to give the point charge distribution as presented in [26]. For the neutron distribution parameters a grid of 35 points covering the ranges $c_{n}=6.28$ to $7.07 \mathrm{fm}$ and $a_{n}=0.35$ to $0.65 \mathrm{fm}$ was selected and the $\left(\gamma, \pi^{0}\right)$ cross section was calculated at each point. These cross sections were smeared with the experimental $q$ resolution and a two-dimensional interpolation between the 35 smeared cross sections was then used to fit the $\left(\gamma \cdot \pi^{0}\right)$ cross sections in Figure 3 and thus extract the best fit values $a_{n}$ and $c_{n}$ for the neutron distribution for each photon energy bin.

Figure 3 also includes model predictions when the $\pi^{0}$ nucleus interaction is not included in the calculation. For the three lower $E_{\gamma}$ bins the effect of the $\pi^{0}$-nucleus interaction is modest in the fitted $q$ range. For the highest bin the differences are significantly larger, probably due to the increase in the $\pi^{0}$ absorption cross section for $\pi^{0}$ energies in the region of the $\Delta$ resonance. The model describes the data over a wide range of $q$ and $E_{\gamma}$.

The best fit neutron skin extracted from the data taking a $2 \mathrm{pF}$ distribution for the nucleon distributions and a proton $2 \mathrm{pF}$ point distribution from Ref. [26] was found to be $\Delta r_{n p}=0.15 \pm 0.03(\text { stat } .)_{-0.03}^{+0.01}$ (sys.) fm. Comparisons of this result with previous measurements are show in Figure 4. The half-height radius and diffuseness of the neutron distribution are determined to be $c_{n}=6.70 \pm 0.03$ (stat.) fm and

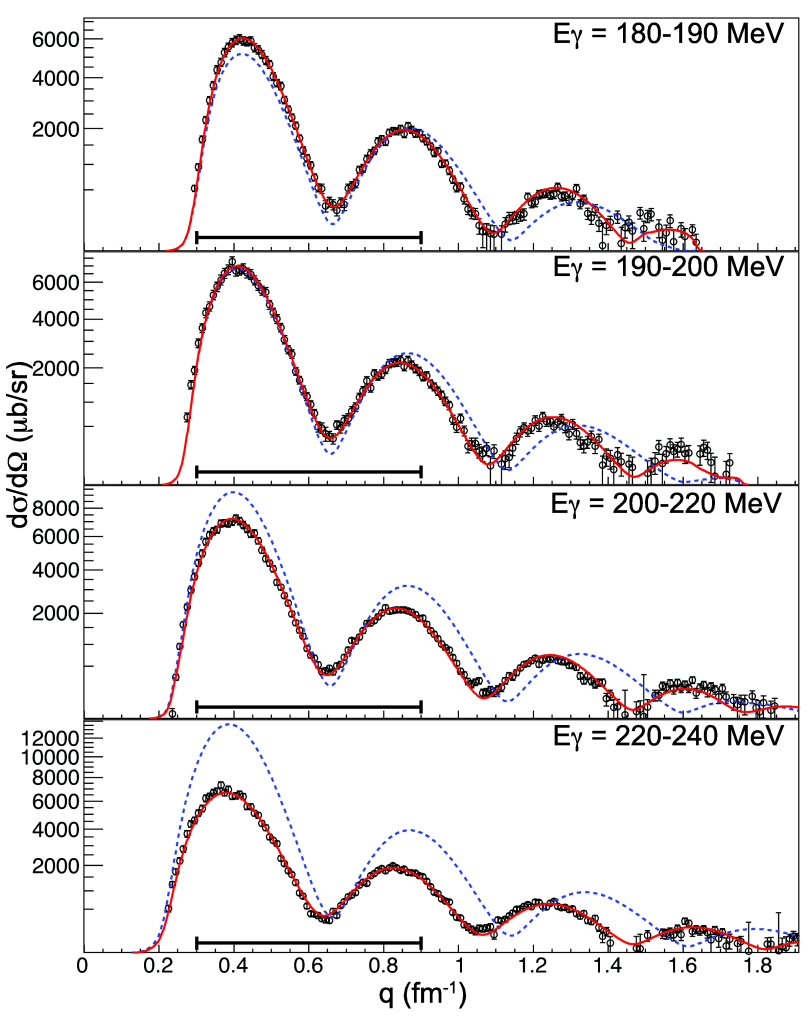

Figure 3. Differential cross sections for the reaction ${ }^{208} \mathrm{~Pb}\left(\gamma, \pi^{0}\right)^{208} \mathrm{~Pb}$ (black circles) for the $E_{\gamma}$ regions indicated. The $\mathrm{y}$-axis employs a square root scale to improve the clarity. The red solid line shows the interpolated fit of the theoretical model to the data. The $q$ range of the fit is indicated by the horizontal bar. The dashed blue line shows the model predictions without including the pion-nucleus interaction.

$a_{n}=0.55 \pm 0.01(\text { stat } .)_{-0.03}^{+0.02}($ sys. $)$ fm respectively. For more details and comparisons to theoretical predictions please see Ref. [30].

Future analysis using more detailed shapes for the nucleon distributions would require improvements to the theoretical model. These are in progress.

\subsection{Further measurements and future plans}

Experimental data is currently under analysis for a chain of tin isotopes ${ }^{116} \mathrm{Sn},{ }^{120} \mathrm{Sn}$ and ${ }^{124} \mathrm{Sn}$. The evolution of the neutron skin over a range of isotopes gives valuable new information on nuclear structure theories and the equation of state e.g. [5] (see Figure 5).

The data analysis is currently at an early stage. The ratios of the $\pi^{0}$ yield for ${ }^{116} \mathrm{Sn}$ and ${ }^{124} \mathrm{Sn}$ are shown in Figure 6 as a function of the polar angle of the $\pi^{0}$. Identical matter form factors for the 2 nuclei would show a flat ratio. The variations in the ratio reflect the differences in the form factors between the nuclei. Once the detector calibrations are complete further detailed analysis will better isolate the coherent yield and information on the difference in the form factors will be extracted from fits to the data. 


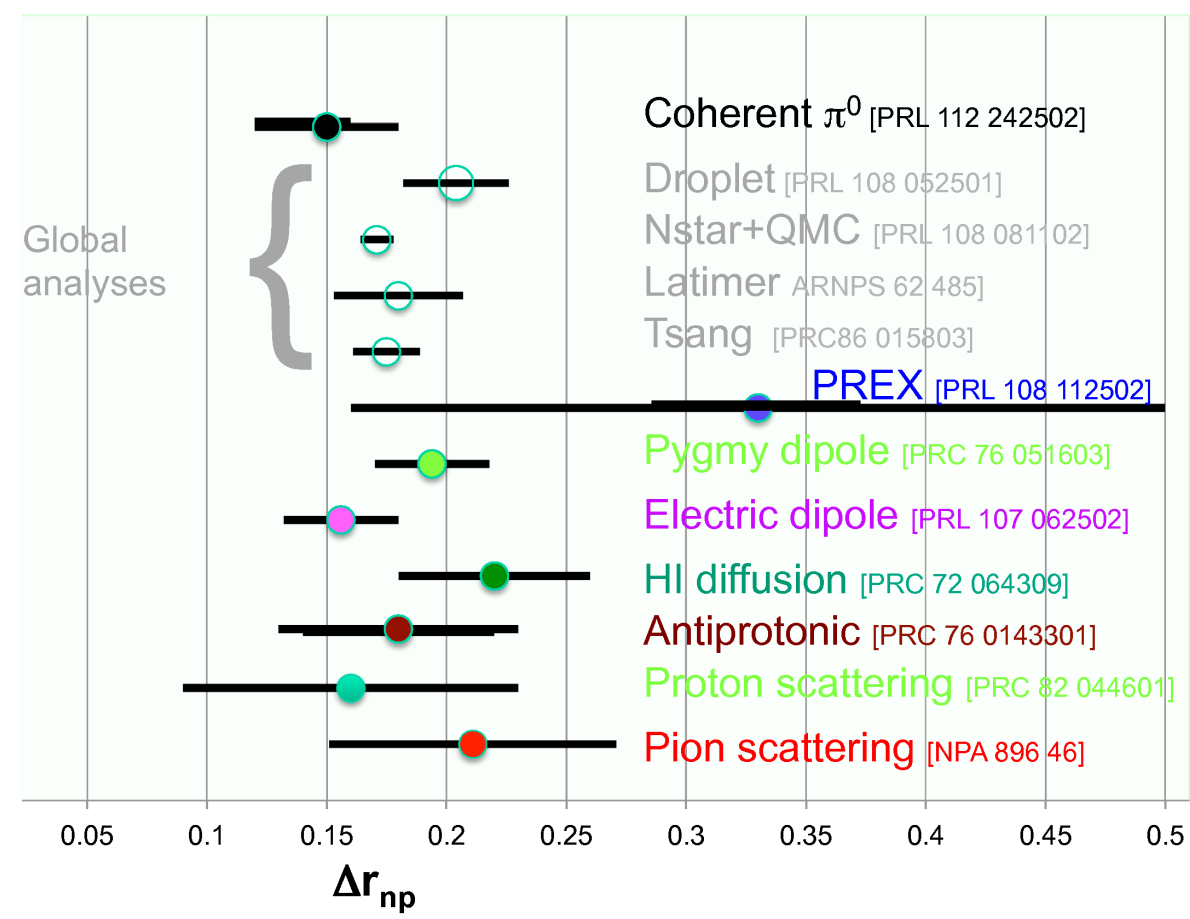

Figure 4. Comparison of this result [30] with previous measurements [13, 14, 19, 20, 26, 31-36]

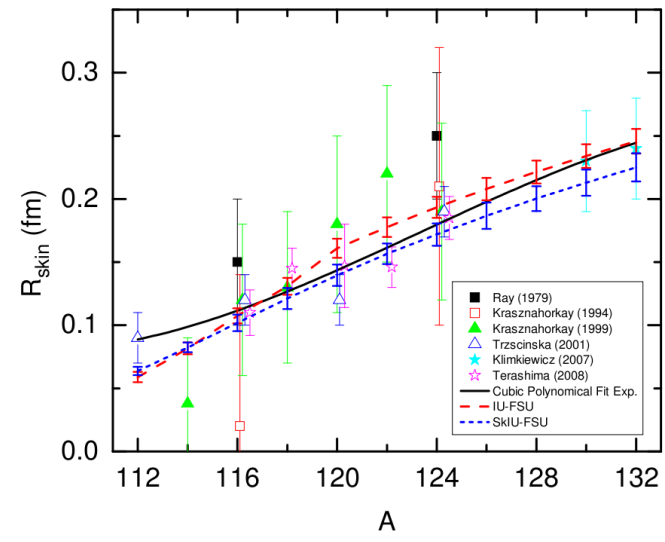

Figure 5. Predictions of the neutron skin for Tin isotopes (lines) and previous experimental data (symbols). The meaning of the different symbols and lines are outlined in Ref. [5].

Data on lighter targets, where the neutron skin should be absent, will also be obtained. The extraction of the coherent yield from light nuclear targets is complicated by the much larger relative contribution of incoherent processes to the yield. These incoherent processes scale as $A$, while the coherent processes scale as $A^{2}$. Nuclear decay- $\gamma$ analysis may offer the opportunity to measure and subtract the incoherent yields over a wide range of $q$ and $E_{\gamma}$. In a previous work we proved this decay- $\gamma$ detection method with the $\mathrm{CB}$ detector and obtained information to constrain the matter form factors for the excited states of ${ }^{12} \mathrm{C}$ [29].

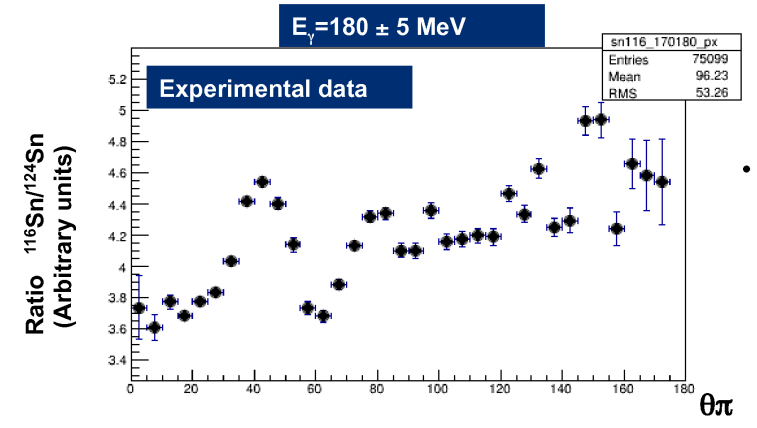

Figure 6. Very early stage analysis of the new tin isotope data from MAMI (see text).

\section{Conclusion}

The coherent photoproduction of $\pi^{0}$ from nuclei is a promising method to extract accurate information on the nature of neutron skins in nuclei. Over the next five years MAMI will produce a number of data sets which will provide valuable, new constraints on the nature of neutron skins to challenge nuclear structure theories and our understanding of the equation of state for nuclear matter.

\section{References}

[1] M. Gmitro, S.S. Kamalov and R. Mach, Phys. Rev. C36 1105 (1987).

[2] B. G. Todd-Rutel and J. Piekarewicz Phys. Rev. Lett. 95, 122501 (2005). 
[3] B.A. Brown Phys. Rev. Lett. 85, 5296 (2000).

[4] S. Typel and B.A. Brown Phys. Rev. C 64, 027302 (2001).

[5] Fattoyev, F. J. and Newton, W. G. and $\mathrm{Xu}$, Jun and Li, Bao-An, Phys. Rev. C86 025804 (2012).

[6] A.W. Steiner, M. Prakash, J.M. Lattimer and P.J. Ellis, Phys. Rep. 411, 325 (2005).

[7] C.J. Horowitz and J. Piekarewicz, Phys. Rev. Lett. 86, 5647 (2001).

[8] J. Xu et. al., Astrophys. J. 697, 1549 (2009).

[9] A.W. Steiner, J.M. Lattimer, and E.F. Brown, Astrophys. J. 722, 33 (2010).

[10] B.G. Todd-Rutel and J. Piekarewicz, Phys. Rev. Lett. 95, 122501 (2005).

[11] De-Hua Wen, Bao-An Li, and Lie-Wen Chen, Phys. Rev. Lett. 103, 211102 (2009).

[12] S.J.Pollock and M.C. Welliver, Phys. Lett. B464 177 (1999)

[13] M.B. Tsang et. al., Phys. Rev. C 86, 015803 (2012).

[14] L. W. Chen, C. M. Ko, and B. A. Li, Phys. Rev. C 72, 064309

[15] K. Hebeler, J.M. Lattimer, C. J. Pethick and A. Schwenk, Phys.Rev. Lett. 105, 161102 (2010)

[16] M. Centelles, X. Roca-Maza, X. Viñas, and M. Warda, Phys. Rev. Lett. 102, 122502 (2009).

[17] A. Carbone et. al., Phys. Rev. C 81, 041301(R) (2010)

[18] L.W. Chen et. al., Phys. Rev. C 82, 024321 (2010).

[19] A. Tamii et. al., Phys. Rev. Lett. 107, 062502 (2011).

[20] J. Zenihiro, H. Sakaguchi, T. Murakami, M. Yosoi, Y. Yasuda, S. Terashima, Y. Iwao, H. Takeda, M. Itoh, H. P. Yoshida, and M. Uchida, Phys. Rev. C 82, 044611 (2010)

[21] B.A. Li, L.W. Chen, and C.M. Ko, Phys. Rep. 464, 113 (2008).

[22] M.B. Tsang et. al., Phys. Rev. Lett. 102, 122701 (2009).

[23] E.D. Bloom and C.W. Peck, Ann. Rev. Nucl. Part. Sci, vol. 33 ,p143(1983)

[24] O. Schwalb et. al, Nucl. Instr. And Meth., A295, p191, (1990); R.Novotny et. al., IEEE Trans Nucl. Sci 38 p379 (1991).
[25] A. Jankowiak et. al., Proc. EPAC2002, Paris (2002).

[26] B. Klos et. al., Phys. Rev. C 76, 014311 (2007).

[27] I. Anthony, J.D. Kellie, S. J. Hall, G. J. Miller and J. Ahrens, Nucl. Instr. Meth. A301, 230 (1991) and S.J. Hall, G.J. Miller, R. Beck and P. Jennewein, Nucl. Instr. Meth. A368 698 (1996).

[28] A. Reiter et. al., http://wwwa2.kph.unimainz.de/microscope/ .

[29] C.M. Tarbert et. al., Phys. Rev. Lett 100, 132301 (2008).

[30] C.M. Tarbert, D.P. Watts, D.I. Glazier et al. Phys. Rev. Lett. 112, 242502 (2014)

[31] Möller, Peter and Myers, William D. and Sagawa, Hiroyuki and Yoshida, Satosh Phys. Rev. Lett. 108, 052501 (2012)

[32] Steiner, A. W. and Gandolfi, S. Phys. Rev. Lett. 108, 081102 (2012)

[33] James M. Lattimer Annu. Rev. Nucl. Part. Sci. 62 485 (2012)

[34] S. Abrahamyan et. al., Phys. Rev. Lett. 108, 112502 (2012)

[35] Klimkiewicz, A. et al. Phys.Rev. C76 (2007) 051603

[36] E. Friedman, Nucl. Phys. A 89646 (2012).

[37] D. Watts, in Proc. of the 11th Int. Conf. on Calorimetry in Part. Phys., Perugia, Italy 2004, (World Scientific, Singapore, 2005, p. 560).

[38] P. Drexler et. al., IEEE Trans Nucl. Sci. 50 p969 (2003).

[39] R.A. Schrack et. al., Phys. Rev. 1271772 (1962).

[40] B. Krusche, Eur. Phys. J. A26 7 (2005).

[41] G. Audit et. al., Nucl. Instr. Methods A, 301 (1991), p. 473.

[42] D. Drechsel et. al., Nucl. Phys. A645 145 (1999).

[43] To check the validity of approximating $\rho(r)$ by a single $2 \mathrm{pF}$ distribution, the Fourier transforms of $\rho(r)$ and the fitted $2 \mathrm{pF}$ distribution were compared. In the momentum range up to $q=0.9 \mathrm{fm}^{-1}$, over which the $\left(\gamma, \pi^{0}\right)$ cross section was fitted to extract information about $\rho_{n}(r)$, the fractional difference between the two transforms only rises to $0.3 \%$ at $q=0.9 \mathrm{fm}^{-1}$.

[44] M. Warda, X. Viñas, X. Roca-Maza, and M. Centelles, Phys. Rev. C 81, 054309 (2010). 